\title{
TRAITEMENT D'UN CAS DE DEBAB DU DROMADAIRE PAR LE BAYER 205
}

\author{
Par L. HERZOG et G. LAVIER
}

Après avoir examiné le sang d'une cinquantaine de dromadaires des souks de Marrakech, le 24 août 1922, nous pûmes en rencontrer un atteint de Debab. Il s'agissait d'une chamelle âgée de 12 ans environ, extrêmement maigre (poids approximatif : $350 \mathrm{~kg}$.) et dans un état de misère physiologique accentuée.

A l'examen direct du sang, on rencontrait par champ (Stiassnie, obj. 6) une trentaine de trypanosomes. L'animal fut hospitalisé au Service de l'Elevage. Le soir même, 4 chiens furent inoculés dans le péritoine avec $5 \mathrm{cc}$. de son sang citraté.

Le 25 août, la chamelle reçut dans la jugulaire 4 gr. de Bayer 205, en solution à 10 p. 100 , elle supporta très bien l'injection et ne montra pas la moindre réaction. Le 26 août, à l'examen direct du sang, les trypanosomes ont disparu. Depuis, l'état général de l'animal s'est grandement amélioré ; à la date du 21 février 1923, il se portait fort bien ; les chiens inoculés chaque mois depuis septembre 1922 avec son sang n'ont jamais montré de trypanosomes.

Les premiers chiens inoculés et non traités moururent en 58 à 65 jours. Une chienne inoculée à partir de l'un d'eux présenta une quarantaine de jours après, des oscillations thermiques (autour de $39^{\circ} 6$ ) et une kératite interstitielle double. Elle reçut le $42^{\circ}$ jour une injection intraveineuse de 50 centigr. de Bayer 205. Après 48 heures, les trypanosomes avaient disparu du sang circulant; au cours de la semaine, la température revint à la normale et les symptômes oculaires disparurent.

On est en somme autorisé à croire à la guérison de notre chamelle. C'est là un résultat important au point de vue pratique. En effet, l'emploi du 205, même en une seule injection, permettrait de stériliser les animaux qu'il est pratiquement impossible, en milieu indigène, de revoir à la visite. Ce médicament a de plus l'avantage de pouvoir être utilisé par la voie sous-cutanée sans produire d'accidents ; il n'en est pas de même avec les autres produits dont la pureté est souvent douteuse (orpiment), le dosage

Annales de Parasitologie, t. $1, \mathrm{~N}^{\circ} 1 .-$ Avril. 1923. 
difficile ou la technique d'injection délicate (émétique, qui produit des nécroses ou des phlébites si le produit n'est pas rigoureusement mis dans la veine). Un contrôle du " Debab " du chameau complété par l'injection de Bayer 205 à tous les animaux reconnus malades pourrait ainsi amener la disparition de l'épizootie et peut-être par contre-coup celle du "Debab " du cheval, si l'on admet la parenté de ces deux affections.

Laboratoire du Service de l'élevage à Marrakech et Lajoratoire de Parasitologie de la Faculté de médecine de Paris. 